\title{
Long-Term Therapy to Prevent Stroke
}

\author{
Howard S. Kirshner, MD, José Biller, MD, and Alfred S. Callahan III, MD
}

Cerebrovascular disease is the third leading cause of mortality and the leading cause of long-term neurological disability in the United States. Most strokes are of ischemic origin and, other than cardioembolic or small vessel strokes, are caused by the development of platelet-fibrin thrombi on an atherosclerotic plaque. This underlying disease mechanism shares important features with coronary artery disease and peripheral artery disease, highlighting the systemic nature of atherothrombosis and the elevated cross risk in stroke patients for ischemic events in other vascular beds. It has been estimated that up to $80 \%$ of ischemic strokes could be prevented with application of currently available treatments for blood pressure, cholesterol, and antithrombotic therapies. Stroke is not, like cancer, waiting for a scientific breakthrough; stroke preventive treatments are well understood and widely available. It is only the application of these treatments to patients, many of whom do not visit physicians, that is lacking. Clearly, better education of the public and active participation of primary care physicians is essential to get the message out to all those at risk. (J Am Board Fam Pract 2005;18:528-40.)

Current treatment strategies for ischemic stroke center around the management of modifiable risk factors through a combination of lifestyle modifications, including diet, exercise, smoking cessation, carotid artery surgery in high-risk patients, and pharmacologic treatment with antihypertensive, antihyperlipidemic, anticoagulant, and/or antiplatelet agents. Anticoagulation is indicated definitely only for patients with atrial fibrillation, less definitely in a few other conditions. Antiplatelet

Submitted 8 February 2005; revised 8 June 2003; accepted 21 June 2005

From the Department of Neurology, Vanderbilt University Medical Center, Nashville, TN (HSK); Department of Neurology, Loyola University Chicago, Stritch School of Medicine, Maywood, IL (JB); and Neurologic Consultants P.C., Nashville, TN (ASC).

Funding: JB has received research grants from National Institutes of Health, Yamanouchi, Boehringer Ingelheim, Centocor, and Astra Zeneca and is on the speakers bureau for Sanofi/BMS. ASC has received research grants from Sanofi, Pfizer, and BMS, is a consultant to Pfizer, serves on the steering committee for SPARCL (Pfizer), and is on the speakers bureau for: BI, Sanofi/BMS, and Pfizer. HSK has received research grants from National Institutes of Health, Sanofi Synthelabo, Boehringer Ingelheim, Pfizer, ONO, Fujisawa, and Aventis, is a consultant to Sanofi SynthelaboBristol Myers Squibb partnership, Wyeth, Merck, Astra Zeneca, and Novartis, and is on the speakers bureau for Boehringer Ingelheim, Sanofi Synthelabo-BMS partnership, Wyeth, Astra Zeneca, Pfizer, Novartis, Janssen, and Forest.

Conflict of interest: none declared.

Corresponding author: Howard S. Kirshner, MD, Department of Neurology, Vanderbilt University School of Medicine, 2100 Pierce Avenue, Nashville, TN 37212 (e-mail: Howard.Kirshner@vanderbilt.edu). therapies that have shown the most promise in secondary stroke prevention include aspirin, clopidogrel, and low-dose aspirin plus extended-release dipyridamole. Given the ongoing risk of secondary atherothrombotic events in stroke survivors, the continuum of care from hospital discharge to the primary care setting must ensure that secondary prevention measures are initiated early and maintained long-term.

Cerebrovascular disease is the third leading cause of death in the United States, accounting for over 163,000 deaths in $2001 .^{1}$ Of those who survive, $40 \%$ recover with moderate to severe neurological impairment; $25 \%$ recover with minor impairment, and only $10 \%$ achieve complete recovery. ${ }^{2}$ Stroke is the leading cause of neurological disability in adults.

Approximately $85 \%$ of strokes are ischemic in origin. ${ }^{2}$ Atherothrombosis, which can be defined as the formation of an arterial thrombus on a background of atherosclerosis, is regarded as the mechanism underlying progression of atherosclerotic disease to an acute ischemic event. The underlying pathophysiology of atherothrombosis is common to coronary artery disease (CAD), peripheral artery disease (PAD), and cerebral ischemic stroke/transient ischemic attack (TIA), at least those TIAs and strokes secondary to large vessel atherothrombosis. In stroke, carotid plaques are the cause of large vessel strokes, and both plaque rupture and inflam- 
mation are important causative factors. $\left.{ }^{3}\right)$. Compared with $\mathrm{CAD}$, stroke is characterized by a diversity of pathophysiologies that have no corresponding type in CAD, eg, lacunar stroke.

Stroke survivors are at increased risk of recurrent stroke ${ }^{2}$ and other ischemic events. In a retrospective study of 1631 stroke survivors, the cumulative incidence of secondary stroke, acute myocardial infarction (MI), and vascular deaths increased over time: $4.2 \%, 6.5 \%, 9.8 \%$, and $11.8 \%$, at $0.5,1,2$, and 3 years, respectively. ${ }^{4}$ There is evidence from major clinical trials ${ }^{5,6}$ that recurrent stroke is the most likely next event after an initial stroke. Stroke is also associated with substantial long-term mortality: 10-year mortality was reported to be $79 \%$, with the main causes of death being sequelae of the initial stroke $(27 \%)$ and cardiovascular disease $(26 \%){ }^{7}$ Patients presenting with ischemic stroke in this study were twice as likely to die from cardiovascular disease as from recurrent stroke, suggesting that coronary death is increasingly important in the longer term.

Stroke should be considered an eminently preventable disease, in terms of the known risk factors. Gorelick and colleagues ${ }^{8}$ estimated that up to $80 \%$ of strokes could be prevented with currently available treatments such as antihypertensive, lipid-lowering, and antithrombotic therapy. Given the risk of secondary atherothrombotic events and associated mortality, it is critically important that stroke survivors be maintained on long-term treatment. The transition to primary care after hospitalization should be managed carefully to ensure continuity of care, and effective communication between the neurologist and primary care physician is paramount. Unfortunately, a substantial proportion of patients are discharged without appropriate antiplatelet or anticoagulant therapy: a recent study showed that $>40 \%$ of patients hospitalized with TIA or stroke were discharged without antithrombotic therapy. ${ }^{9}$ In a review of stroke prevention, Holloway and colleagues ${ }^{10}$ estimated $53 \%$ to $80 \%$ adherence to discharge of stroke patients on antithrombotic therapy, $27 \%$ to $44 \%$ on antihypertensive therapy, and $67 \%$ with documented smoking cessation counseling. In the 4 intial Coverdell states' reporting, ${ }^{11} 89 \%$ of patients were discharged on antiplatelet therapy, but only $40 \%$ of atrial fibrillation patients were discharged on warfarin. Clearly, greater adherence to guidelines is needed. Hospital-based stroke prevention pro- grams such as UCLA Medical Center's Stroke PROTECT (Protecting Recurrence of Thromboembolic Events through Coordinated Treatment) program, which integrates secondary prevention measures into the standard stroke care provided during hospitalization, have proved effective at improving antithrombotic, angiotensin-converting enzyme (ACE) inhibitor, and 3-hydroxy-3-methylglutaryl coenzyme A (HMG-CoA) reductase inhibitor ("statin") utilization rates at discharge. ${ }^{12} \mathrm{By}$ facilitating the in-hospital initiation of secondary prevention measures, such programs should also improve long-term treatment rates and clinical outcomes. Overall, the utility of these programs highlights the importance of a systems approach to quality improvement, rather than an ad hoc approach to stroke prevention.

Another important aspect of preventive care is the adherence of stroke patients themselves to therapy. Some patients may be reluctant to continue on their treatment protocol long-term, especially those who lack insurance drug benefits. Primary care physicians are in a position to monitor and support such patients.

\section{Cross-Risk Factors for Atherothrombotic Stroke}

Because atherothrombosis is the underlying mechanism common to $\mathrm{CAD}, \mathrm{PAD}$, and cerebral ischemic stroke/TIA, it is not surprising that there is a degree of cross-risk between these conditions (Figure 1). For example, a 10-year prospective study found that patients with large-vessel PAD were at 3.1-fold greater risk of all-cause mortality, 5.9-fold greater risk of cardiovascular mortality, and 6.6fold greater risk of coronary heart disease than subjects without evidence of the disease. ${ }^{13}$

Studies in patients with ischemic stroke have also highlighted the coexistence of symptomatic atherothrombotic conditions. It is estimated that

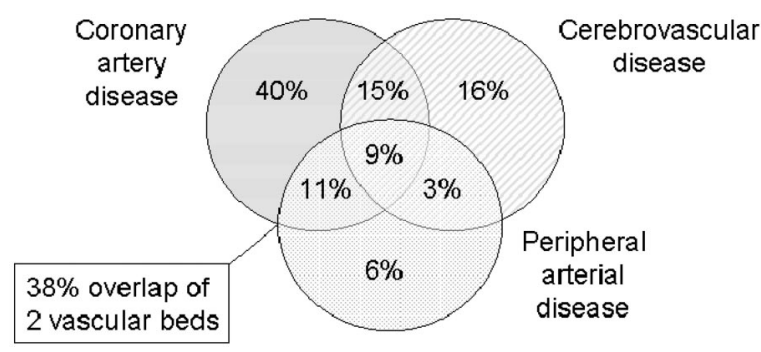

Figure 1. Overlap of atherosclerotic disease. ${ }^{14}$ 
$20 \%$ to $40 \%$ of stroke patients may have abnormal tests for silent myocardial ischemia, and that $25 \%$ to $60 \%$ of patients with carotid disease and no overt CAD have abnormal tests for myocardial ischemia or angiographic evidence of severe CAD.${ }^{14} \mathrm{~A}$ study in geriatric patients with ischemic stroke showed that $\mathrm{CAD}$ was also present in $56 \%$ of patients and $\mathrm{PAD}$ was present in $28 \%$; conversely, stroke was present in $32 \%$ of CAD and $42 \%$ of PAD cases. ${ }^{15}$ In addition, the occurrence of a TIA has been identified as a risk factor for other ischemic events, as indicated by the high incidence (25\%) of recurrent TIA, stroke, vascular hospitalization, or death in the 90 -day period after a TIA. ${ }^{16}$ Immediate evaluation of TIA patients is crucial, with evaluation of risk factors and exploration of the mechanism of the TIA so that preventive treatments can be started immediately.

\section{Pathophysiology of Stroke}

Atherosclerosis is characterized by the accumulation of lipids, cellular waste products, calcium, and other substances in the inner arterial lining. Plaque formation occurs by the passive diffusion of lipid (low density lipoprotein [LDL]) into the subendothelial space. Only rarely do patients have an effective barrier at the endothelial cell to the passage of lipid. As lipid accumulates, plaques can become unstable when their caps are thinned, leading to rupture at the plaque shoulders. When atherosclerotic plaques rupture, a thrombus forms, which can interrupt blood flow or break off and embolize to another part of the body. Recent evidence affirms the mechanism of platelet activation to atherogenesis and the crucial role of this process in the pathogenesis of atherothrombotic stroke. Inflammation in plaques also plays an important role, and C-reactive protein seems to be an independent risk factor for stroke. ${ }^{17,18}$ When the atherosclerotic plaque is disrupted, platelets adhere to the damaged endothelium and undergo activation and aggregation into a platelet-rich thrombus. Increased levels of surrogate platelet activation markers are present after ischemic stroke, indicating increased platelet reactivity. ${ }^{19}$ Although the platelet plays a role in the occlusive event over an activated plaque, artery-toartery embolism is more often the causative event in stroke, rather than local occlusion.

The potential for synergy among antiplatelet agents that target different pathways of platelet aggregation has led to combinations of different antiplatelet agents being investigated for secondary stroke prevention, eg, extended-release (ER)-dipyridamole + aspirin and clopidogrel + aspirin, as described later.

\section{Primary Prevention of Ischemic Stroke}

Prophylactic strategies for ischemic stroke center largely on the management of modifiable risk factors (Table 1). Guidelines recommend that patients adopt healthy lifestyle habits such as avoidance of tobacco (including passive smoking), healthy eating patterns (including consumption of fruits, vegetables, low-fat dairy products, and lean meat), regular appropriate exercise, and minimization of alcohol intake. ${ }^{20}$ Carotid endarterectomy or carotid stenting may be considered for patients with carotid stenosis, at high risk of stroke. ${ }^{21}$

At-risk patients can be treated with ensembles of therapies or pharmacotherapy that may include antihypertensive agents, statins, and antithrombotic therapies. Patients with atrial fibrillation benefit from anticoagulation. In primary prevention in men, aspirin prevents MI but not stroke ${ }^{22}$ (American Physicians Heart study), but in women, there seems to be a reduced risk of stroke as well in both middle aged and elderly subjects. ${ }^{23}$ Use of aspirin for primary prevention in middle aged and elderly people, especially those with cardiovascular risk factors, is a consistent recommendation (" $\mathrm{A}$ " recommendation by the Strength of Recommendation Taxonomy [SORT] criteria $^{24}$ ), as long as both heart attack and stroke are considered as the endpoint.

A full account of effective approaches for the primary prevention of stroke is provided by various recent reviews ${ }^{25,26}$ and consensus/scientific statements. $^{20,25,28}$

Table 1. Modifiable Risk Factors for Stroke $\mathrm{e}^{20,21}$

Risk Factor

Hypertension

Smoking (including passive smoking)

Diabetes

Asymptomatic carotid stenosis

Increased blood lipid levels

Atrial fibrillation (nonvalvular)

Obesity

Physical inactivity

Excess alcohol intake 


\section{Role of Long-Term Therapy for Secondary Prevention of Ischemic Stroke}

Many of the recommended strategies for the primary prevention of ischemic stroke also apply to the secondary prevention of stroke, although antiplatelet therapy is more prominent in the latter setting. Treatment of risk factors such as hypertension, hyperlipidemia, atrial fibrillation and carotid stenosis are important in secondary stroke prevention. $^{26-28,30}$ Smoking cessation is almost certainly of benefit, although this has not been proved by prospective clinical trials.

Updated 2004 guidelines for the management of stroke were published by the National Stroke Association $(\mathrm{NSA})^{29}$ and the American College of Chest Physicians (ACCP). ${ }^{28}$ The NSA rated treatments as "appropriate," "uncertain," or "inappropriate." The ACCP rated treatments based on the strength of recommendation (grade 1 is the strongest) and the level of evidence (eg, ranging from grade A [consistent results from randomized clinical trials] to grade $\mathrm{C}$ [evidence from observational studies]). Where available, we have included the NSA and ACCP recommendations, as well as the SORT criteria, ${ }^{24}$ familiar to readers of the Journal of the American Board of Family Practice.

\section{Antihypertensive Therapy}

Antihypertensive therapy is effective in reducing the risk of recurrent stroke ${ }^{31}$; information on the use of specific antihypertensive agents is provided by the seventh report of the Joint National Committee (JNC7) guidelines. ${ }^{32}$ In the PROGRESS study, ${ }^{33}$ active treatment with perindopril, with or without the diuretic indapamide, reduced the risk of recurrent stroke by $28 \%$. The ACCESS study showed that, compared with placebo, early treatment with candesartan within 24 hours of stroke was associated with fewer vascular events after 1 year $(9.8 \%$ vs $18.7 \%)$ and lower cumulative 12 month mortality $(2.9 \%$ vs $7.2 \%) .{ }^{34}$ These results suggest that the risk of inducing neurological worsening by acute lowering of blood pressure may be overestimated, although very cautious introduction of an antihypertensive agent, in this case candesar$\tan$ at a low dose of $4 \mathrm{mg}$ daily, is the most aggressive acute therapy recommended at present. Careful identification of hypertensive patients with stroke and TIA, with initiation of single or combination therapy, is essential for secondary stroke prevention.
Considerable controversy has surrounded the choice of specific antihypertensive agents for stroke prevention. The PROGRESS ${ }^{33}$ and ACCESS ${ }^{34}$ trials suggest that ACE inhibitors or angiotensin II receptor blockers (ARBs) may be especially effective in secondary stroke prevention. The HOPE trial $^{35}$ also confirmed a benefit of the ACE inhibitor ramipril in preventing strokes, along with myocardial infarction and cardiac death, in patients more than age 55 with additional vascular risk factors. The $\mathrm{LIFE}^{36}$ trial suggested that an ARB (losartan) was superior to a $\beta$-blocker (atenolol) for prevention of stroke. On the other hand, the ALLHAT trial, ${ }^{37}$ a primary trial of antihypertensive therapies, found little difference among the ACE inhibitor, diuretic, and calcium channel arms, and the diuretic had a small advantage overall. There has been increasing evidence that ACE inhibitors alone are not effective treatments in African American patients. ${ }^{38}$ The VALUE study ${ }^{39}$ also showed relative equivalence of a calcium channel blocker, amlodipine, versus an $\mathrm{ARB}$, valsartan, in stroke prevention. A reasonable conclusion, as suggested by the JNC7 recommendations, ${ }^{32}$ is that blood pressure should be controlled in stroke patients, and an algorithm for specific agents is included. In our opinion, therapy should be initiated before discharge from the hospital after a stroke to maximize compliance. The target blood pressure should be below the JNC7 cutoff of $140 \mathrm{~mm} \mathrm{Hg}$ systolic and $90 \mathrm{~mm} \mathrm{Hg}$ diastolic.

The NSA rates treatment with most antihypertensive agents as appropriate; the use of calcium channel blocker alone or with diuretic, or diuretic plus $\beta$-blocker, is rated as uncertain. ${ }^{29}$ Clearly, treatment of hypertension in stroke patients merits an "A" rating by the SORT criteria. ${ }^{24}$

\section{Statin Treatment}

According to the Stroke Council, the vast majority of patients with previous ischemic stroke or TIA are likely to benefit from statin use. ${ }^{40}$ In the subgroup of patients enrolled in the Heart Protection Study $^{41}$ who had cerebrovascular disease but no CAD at study entry, the risk of major vascular events (coronary events, stroke, or revascularization) was reduced by $23 \%$ with $40 \mathrm{mg}$ of simvastatin compared with placebo; there was no reduction in second strokes as a single endpoint. The mean duration of follow-up for this subgroup of patients 
was 4.8 years. The age of the patient and the level of the LDL cholesterol did not influence the results. Based on results of the Heart Protection Study, ${ }^{42}$ the FDA approved simvastatin at a dose of $40 \mathrm{mg}$ daily for secondary prevention in patients with stroke or TIA. The ongoing SPARCL (Stroke Prevention with Aggressive Reduction in Cholesterol Levels) study will indicate whether all patients with TIA or stroke, in the absence of coronary or peripheral vascular disease, should be placed on statin therapy with atorvastatin. ${ }^{43}$ Statin therapy for stroke has not been taken up in guidelines concerning hyperlipidemia, but the evidence is sufficient, in our opinion, to warrant an " $\mathrm{A}$ " recommendation by the SORT criteria. ${ }^{24}$

\section{Anticoagulant Treatment}

Warfarin is effective in the primary prevention of thromboembolic stroke in patients with atrial fibrillation, as evidenced by a meta-analysis showing that warfarin consistently decreased the risk of stroke by $68 \% .{ }^{44}$ Furthermore, in a study investigating secondary prevention of stroke in patients with nonrheumatic atrial fibrillation and recent TIA or minor stroke, warfarin was more effective than aspirin, with 90 versus 40 vascular events (mainly stroke) being prevented each year for every 1000 patients. ${ }^{44}$ No other antithrombotic agents or combinations besides warfarin and aspirin have been studied in this population, although the ACTIVE (atrial fibrillation clopidogrel trial with irbesartan for the prevention of vascular events) trial, comparing aspirin + clopidogrel to warfarin, is ongoing. Recently, the study arm comparing aspirin plus clopidogrel versus warfarin was stopped because of excess strokes in the antiplatelet group. An experimental thrombin inhibitor, ximelagatran, has been tested in atrial fibrillation with results comparable with warfarin, ${ }^{46,47}$ but the FDA has denied approval of this agent based on safety risks.

Results from warfarin studies in cohorts other than atrial fibrillation patients have not demonstrated similar efficacy. For example, a study of warfarin versus aspirin for prevention of recurrent cerebral ischemia of presumed noncardiac origin was terminated because of a high rate of major bleeding complications with warfarin. ${ }^{48}$ The WARSS (warfarin-aspirin recurrent stroke study) trial $^{49}$ reported no significant difference in efficacy between warfarin and aspirin for the prevention of recurrent ischemic stroke in patients with prior noncardioembolic ischemic stroke; there was a greater (nonsignificant) benefit of aspirin over warfarin in males versus females. In a prospective cohort study within the WARSS trial, there was no correlation between risk for a subsequent vascular occlusive event and antiphospholipid antibodies. ${ }^{50}$ The same was true in a substudy involving patients with patent foramen ovale. ${ }^{51}$

Warfarin has also not proved superior to aspirin in secondary prevention of stroke in patients with intracranial artery stenosis. Results from the WASID study showed no efficacy difference between warfarin and aspirin, but the warfarin group suffered more bleeding complications and greater overall mortality. ${ }^{52}$ In addition, a study comparing these agents in stroke patients with valvular strands did not reveal an advantage for warfarin. ${ }^{53}$

Warfarin is used by consensus only in patients with atrial fibrillation or a similar, definite cardiac source of embolus (prosthetic heart valve, MI with mural thrombus, cardiomyopathy or low ejection fraction congestive heart failure, and even the low ejection fraction indication is being studied. Warfarin has also been used by some stroke experts in 3 other situations: (1) cerebral venous sinus thrombosis; (2) acute extracranial internal carotid or vertebral artery dissections; and hypercoagulation states. Use of warfarin in venous sinus thrombosis is supported by small clinical trials and by anecdotal series, but most neurologists recommend this therapy. With regard to dissections of the carotid or vertebral arteries, there is very little clinical trial evidence, and this remains a matter of individual judgment. Routine anticoagulation for all stroke patients, or for treatment failures with antiplatelet therapy, does not seem warranted.

The NSA rates anticoagulation treatment for patients with atrial fibrillation as appropriate (those with low bleeding risk) and uncertain (those with high bleeding risk), and inappropriate for patients without atrial fibrillation. ${ }^{29}$ The ACCP recommends long-term anticoagulation therapy in patients with cardioembolic stroke and underlying atrial fibrillation (grade 1A) and only in noncardioembolic stroke patients with well documented prothrombotic disorders (grade 2C). ${ }^{28}$ Our recommendations, according to the SORT criteria, ${ }^{24}$ would be an "A" rating for warfarin for atrial fibrillation, "B" for all other indications. 


\section{Antiplatelet Treatment}

Antiplatelet therapy plays an important role in the long-term prevention of ischemic stroke and vascular events in patients who have experienced acute ischemic stroke or TIA. ${ }^{26,54,55}$ In a meta-analysis of 287 studies involving patients at high risk for vascular ischemic events, antiplatelet therapy reduced the risk of ischemic stroke by $30 \% .{ }^{55}$ For patients who had experienced previous stroke or TIA, the incidence of nonfatal stroke recurrence was reduced from $10.8 \%$ to $8.3 \%$, corresponding to 25 fewer strokes per 1000 patients treated.

The most commonly used antiplatelet therapies are aspirin, ER-dipyridamole + low-dose aspirin, and the ADP receptor antagonist clopidogrel.

\section{Aspirin}

Aspirin is well established as an effective antiplatelet agent. ${ }^{56}$ In a meta-analysis of trials of aspirin in the secondary prevention of cardiovascular and cerebrovascular events, aspirin reduced the number of strokes by $20 \%{ }^{56}$ However, aspirin therapy is not without controversy. Long-term treatment with aspirin has been associated with adverse bleeding effects (see Safety section). Lower doses of aspirin have been implemented to counteract this problem, but there remains uncertainty about whether such low doses are as effective as daily doses of $\geq 75 \mathrm{mg}$. ${ }^{55,56}$

\section{Aspirin + ER-Dipyridamole}

The ESPS2 (European Stroke Prevention Study 2) compared placebo, $25 \mathrm{mg}$ of aspirin twice a day monotherapy, $200 \mathrm{mg}$ of ER-dipyridamole twice a day monotherapy, and $25 \mathrm{mg}$ of aspirin $+200 \mathrm{mg}$ of ER-dipyridamole in the secondary prevention of stroke. ${ }^{6}$ The combination of aspirin + ER-dipyridamole was effective in reducing the risk of stroke recurrence. Compared with aspirin monotherapy, the combination therapy reduced stroke incidence by $23 \%$. Neither aspirin monotherapy nor aspirin + ER-dipyridamole combination therapy significantly reduced the endpoints of stroke and death, or death alone, compared with placebo, but the combined endpoint of stroke, MI, and vascular death was significantly reduced. A recent report has found an even greater effect of aspirin + ER-dipyridamole in "high risk" patients. ${ }^{56,57}$ The greatest limiting factor in use of aspirin + ER-dipyridamole is the lack of published evidence for an effect in patients with coronary artery or peripheral vascular disease.

\section{Clopidogrel}

In the CAPRIE trial, long-term treatment $(>1$ to 3 years) with clopidogrel in patients with recent ischemic stroke, MI, or PAD ( $\mathrm{n}=19,185)$ was more effective than aspirin in reducing the combined risk of ischemic stroke, MI, or vascular death (relative risk reduction of $8.7 \%$ ). For the subgroup of patients with ischemic stroke, the relative risk reduction was $7.3 \%$, similar to the study population as a whole but not statistically significant for the subgroup. ${ }^{5}$

Post hoc analyses of this trial have shown that the benefits of clopidogrel over aspirin are amplified in certain high-risk populations. For example, in diabetic patients, the annual event rate for stroke, MI, vascular death, or rehospitalization for ischemia or bleeding was $17.7 \%$ for patients treated with aspirin, compared with $15.6 \%$ for those who received clopidogrel (relative risk reduction $11.9 \%) .{ }^{58}$ Similarly, in patients with prior coronary artery bypass surgery, the annual event rate of ischemic stroke, MI, vascular death, or rehospitalization for ischemia was $21.6 \%$ for aspirin and $15.2 \%$ for clopidogrel (relative risk reduction 29.3\%). ${ }^{59}$

Another post hoc analysis of the CAPRIE study investigated a subgroup of patients with a history of $>1$ previous acute ischemic event (ischemic stroke or MI). ${ }^{60}$ Compared with the overall population, these patients had an increased risk for the endpoints studied (ischemic stroke, MI, or vascular death and ischemic stroke, MI, and rehospitalization for ischemia) (Figure 2). Over 3 years, the number needed to treat to prevent one ischemic stroke, MI, or vascular death was 91 for the overall CAPRIE study population, compared with only 29 for this subgroup. Interestingly, this number might reflect the average number of patients seen by a primary care physician daily.

\section{Clopidogrel + Aspirin}

The superiority of clopidogrel over aspirin found in previous studies provided the rationale for the MATCH study, which investigated whether the addition of aspirin to clopidogrel would confer additional benefit. $^{61}$

This study involved patients who had experienced an ischemic stroke or TIA in the previous 3 months (mean 27 days) and had at least one other 


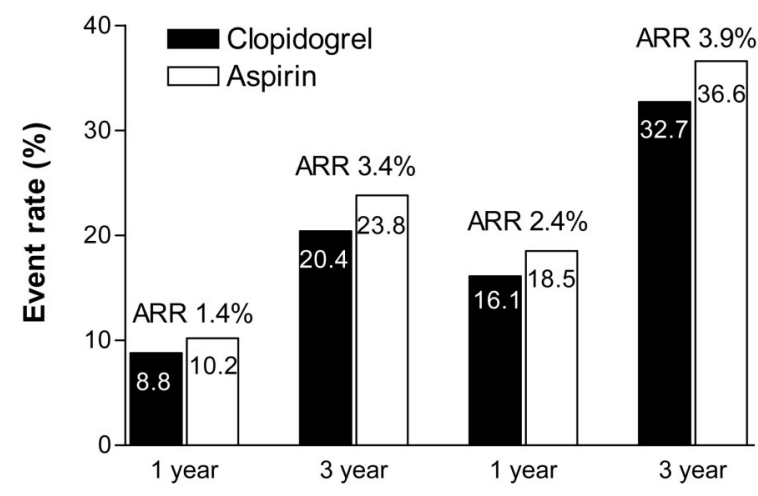

IS, MI, VD

IS, MI, Rehospitalization

Figure 2. Efficacy of long-term clopidogrel or aspirin therapy in patients with $>1$ previous acute ischemic event based on 1-year and 3-year event rates for 2 composite endpoints. ${ }^{45}$ The overall relative risk reduction for clopidogrel versus aspirin was 14.9\% ( $P=.045)$ for the endpoint of ischemic stroke (IS), myocardial infarction (MI), and vascular death (VD) and $12.0 \%(P=.039)$ for IS, MI, and rehospitalization.

risk factor such as previous $\mathrm{MI}$ or stroke, angina, diabetes, or symptomatic PAD. The MATCH population included $68 \%$ diabetic patients, $54 \%$ lacunar strokes, and 10\% strokes of unknown origin. Only 30\% of the patients had large vessel atherosclerotic disease. The MATCH study compared 75 $\mathrm{mg}$ of clopidogrel once a day monotherapy with the combination of $75 \mathrm{mg}$ of aspirin $+75 \mathrm{mg}$ of clopidogrel once a day.

For the primary endpoint (ischemic stroke, MI, vascular death, or rehospitalization for an acute ischemic event), the addition of aspirin to clopidogrel did not provide any additional benefit (event rates were $16 \%$ for aspirin + clopidogrel and 17\% for placebo + clopidogrel; relative risk reduction $6.4 \%, \mathrm{NS})$. Furthermore, the addition of aspirin to clopidogrel resulted in a significantly greater number of life-threatening and major bleeding events (see Safety section). For high-risk patients with lacunar stroke, clopidogrel monotherapy seems more advisable than combination aspirin and clopidogrel therapy. It is possible that combination therapy may still have advantages in some stroke patients, such as those with large vessel, atherothrombotic disease ${ }^{62}$ or intracranial stenosis, but specific research on these indications is lacking.

The CARESS study investigated short-term treatment with clopidogrel + aspirin $(75 \mathrm{mg})$ for the reduction of silent cerebral microemboli in a small series of patients with symptomatic carotid stenosis $(>50 \%)$ with TIA or stroke within the last 3 months. ${ }^{63}$ At day 7 , there was a significant reduction in silent cerebral microemboli in favor of clopidogrel + aspirin versus aspirin alone (relative risk reduction $37.3 \%$ ). Furthermore, there was no increase in bleeding events for clopidogrel + aspirin compared with aspirin alone.

Taken together, the results of the MATCH and CARESS studies suggest that, although combination therapy with clopidogrel + aspirin may ultimately be shown to provide benefit for some stroke patients, clopidogrel monotherapy is preferable for the general group of high-risk stroke patients. Moreover, the findings from CAPRIE indicate that the benefits of clopidogrel persist over the longterm (maximum follow-up period 3 years). The timing of clopidogrel + aspirin treatment onset may also be clinically relevant. Evidence from the CURE (clopidogrel in unstable angina to prevent recurrent events) trial, ${ }^{64}$ involving patients with non-ST-segment elevation acute coronary syndromes, showed that early treatment (within 24 hours of the index event) with clopidogrel + aspirin reduced the combined risk of cardiovascular death, nonfatal MI and stroke at day 30, compared with aspirin alone (relative risk $0.79 ; P=.003$ ). Whether early clopidogrel + aspirin treatment is beneficial for some stroke patients is the subject of the FASTER trial (Table 2). This table also details several other studies involving clopidogrel combination therapies that are currently in progress.

For the secondary prevention of stroke, the NSA rates aspirin, aspirin + ER-dipyridamole, and clopidogrel as appropriate, and aspirin + clopidogrel as uncertain; for those patients taking aspirin at the time of their event, aspirin therapy is rated as uncertain, aspirin + ER-dipyridamole, clopidogrel and aspirin + clopidogrel as appropriate. For all patients, the NSA rates ticlopidine (another ADP receptor antagonist) as inappropriate. ${ }^{29}$ In patients who have experienced a noncardioembolic stroke/ TIA, the ACCP recommends antiplatelet therapy with either $75 \mathrm{mg}$ of clopidogrel daily, 50 to 325 $\mathrm{mg}$ of aspirin daily, or $25 \mathrm{mg}$ of aspirin $+200 \mathrm{mg}$ of ER-dipyridamole twice daily. ${ }^{28}$ The ACCP also suggests that both aspirin + ER dipyridamole (2A recommendation) and clopidogrel (2B recommendation) are superior to aspirin alone. By the SORT criteria, $^{24}$ antiplatelet therapy is clearly recom- 


\begin{tabular}{|c|c|}
\hline Trial & Details \\
\hline CHARISMA & $\begin{array}{l}\text { Investigating the effects of adding clopidogrel } 75 \mathrm{mg} / \text { day to low-dose aspirin ( } 75 \text { to } 162 \mathrm{mg} \text { ) in patients } \\
\text { at high risk of developing atherothrombotic events. }{ }^{49} \text { Completed in July } 2005 \text {; results pending. }\end{array}$ \\
\hline \multirow[t]{2}{*}{ SPS3 } & $\begin{array}{l}\text { Investigating the effects of aspirin } 325 \mathrm{mg} / \text { day or aspirin } 325 \mathrm{mg} / \text { day }+ \text { clopidogrel } 75 \mathrm{mg} / \text { day in the } \\
\text { prevention of secondary stroke, major vascular events, and cognitive decline among patients with } \\
\text { small subcortical strokes. }\end{array}$ \\
\hline & $\begin{array}{l}\text { This study is also comparing a standard (130 to } 149 \mathrm{~mm} \mathrm{Hg} \text { systolic) blood pressure against an } \\
\text { aggressive }(<130 \mathrm{~mm} \mathrm{Hg} \text { systolic) blood pressure target, and it is evaluating cognitive status in } \\
\text { addition to recurrent stroke as an endpoint. }\end{array}$ \\
\hline PRoFESS & $\begin{array}{l}\text { This study compares clopidogrel }( \pm \text { telmisartan }) \text { therapy with aspirin }+ \text { dipyridamole }( \pm \text { telmisartan }) \\
\text { for secondary stroke prevention. }\end{array}$ \\
\hline \multirow[t]{2}{*}{ FASTER } & $\begin{array}{l}\text { The trial will involve patients randomized to receive clopidogrel (or placebo) and simvastatin (or } \\
\text { placebo) within } 12 \text { hours of onset of TIA or minor stroke; all patients will receive aspirin. }\end{array}$ \\
\hline & $\begin{array}{l}\text { The only clinical stroke trial to include a loading dose of clopidogrel, as was used in the CURE acute } \\
\text { coronary syndrome trial. }\end{array}$ \\
\hline
\end{tabular}

*Updates on these trials are available online at: http://www.strokecenter.org/trials.

mended for secondary stroke prevention, using aspirin, aspirin + ER-dipyridamole, or clopidogrel.

\section{Safety of Long-Term Antiplatelet Therapy Aspirin \pm Dipyridamole}

Long-term aspirin treatment is associated with an increased risk of hemorrhagic stroke, major intracranial bleeds, and upper gastrointestinal bleeding. ${ }^{56}$ In a recent study involving almost 19,000 patients admitted to hospital with adverse drug reactions, aspirin was the most commonly implicated culprit: $18 \%$ of admissions because of an adverse drug reaction were attributed to aspirin; the majority of these patients were receiving lowdose aspirin $(75 \mathrm{mg} /$ day $){ }^{65}$

Further evidence that low-dose aspirin does not eliminate the propensity for induced bleeding comes from the ESPS2 trial, ${ }^{6}$ which found that all-site bleeding and gastrointestinal bleeding were significantly more frequent in patients receiving aspirin monotherapy $(50 \mathrm{mg} /$ day $)$ than in those receiving ER-dipyridamole $(400 \mathrm{mg} /$ day) or placebo. The combination of aspirin + ER-dipyridamole in this study was associated with a higher incidence of side effects such as headache $(38 \%$ vs $33 \%$; the withdrawal rates caused by this adverse event were $8.1 \%$ vs $1.9 \%$ ) and gastrointestinal disorders (33\% vs 30\%). Life-threatening bleeding was insignificantly more common with aspirin + ER-dipyridamole, compared with aspirin alone (1.6\% vs $1.2 \%)$.

\section{Clopidogrel}

Clopidogrel has a similar safety profile to aspirin in patients at risk of recurrent ischemic events, although it is associated with lower rates of gastric and central nervous system (CNS) adverse events, including gastrointestinal and intracranial hemorrhage (Table 3). This favorable tolerability of clopidogrel is demonstrated even when treatment is given for 1.5 to 3 years, as reported in the CAPRIE and MATCH studies. Cases of thrombotic thrombocytopenic purpura/hemolytic uremic syndrome (TTP/HUS) with clopidogrel use have been reported, but this association has been questioned as alternative causes cannot be excluded. ${ }^{66}$

\section{Clopidogrel + Aspirin}

In the MATCH study, adding aspirin to clopidogrel in high-risk patients with recent ischemic

Table 3. Incidence of Adverse Events in Patients Treated with Clopidogrel or Aspirin ${ }^{4}$

\begin{tabular}{lcc}
\hline & \multicolumn{2}{c}{$\begin{array}{c}\text { Adverse Event } \\
\text { (Percentage of Patients) }\end{array}$} \\
\cline { 2 - 3 } & Clopidogrel & Aspirin \\
\hline Rash & 6.0 & $4.6^{*}$ \\
Diarrhea & 4.5 & $3.4^{*}$ \\
Indigestion/nausea/vomiting & 15.0 & $17.6^{*}$ \\
Any bleeding disorder & 9.3 & 9.3 \\
Intracranial hemorrhage & 0.4 & 0.5 \\
Gastrointestinal & 2.0 & $2.7^{*}$ \\
$\quad$ hemorrhage & & \\
Abnormal liver function & 3.0 & $3.2^{*}$ \\
\hline
\end{tabular}

*Statistically significant $(P<.05)$ 
stroke or TIA resulted in significantly more life threatening (2.6\% vs $1.3 \%)$ and major (2\% vs $1 \%)$ bleeding complications; the rate of intracranial hemorrhage was doubled with clopidogrel + aspirin treatment $(0.9 \%$ vs $0.4 \%) .{ }^{61}$ In the $\mathrm{CURE}^{64}$ trial of patients with acute coronary syndrome, the addition of clopidogrel to aspirin resulted in a $1 \%$ absolute, 34\% relative increase in the risk of major bleeding (life threatening bleeding, substantially disabling bleeding, or bleeding necessitating transfusion of $\geq 2$ units) over the (mean) 9-month treatment period, but there was no increase in lifethreatening bleeding. The bleeding risk was largely dictated by the dose of aspirin: the incidence of major bleeding with high-dose aspirin (200 to 325 $\mathrm{mg}$ ) alone $(3.7 \%)$ was greater than that with the combination of low-dose aspirin (75 to $100 \mathrm{mg}$ ) + clopidogrel (3.0\%), and combined acetylsalicylic acid (ASA) 200 to $325 \mathrm{mg}+$ clopidogrel had a $4.9 \%$ bleeding risk. ${ }^{67}$ In patients with percutaneous stents, ASA, and clopidogrel in combination are the most effective treatment, and the bleeding risk of combination therapy over the 4-week recommended period is no greater than that of aspirin alone. $^{68}$

\section{Carotid Endarterectomy versus Angioplasty and Stenting}

Three studies, all published in 1991, provided evidence of the beneficial effect of carotid endarterectomy in the prevention of cerebral ischemia in symptomatic carotid artery stenosis of greater than $70 \% .{ }^{69-71}$ The efficacy of this procedure was subsequently demonstrated in patients with asymptomatic carotid artery stenosis, ${ }^{72}$ with greater benefit in men than women (reduction in 5-year event rate: $66 \%$ vs $17 \% ; P=.10$ ). Similar benefit was also seen in the recent Asymptomatic Carotid Surgery $\mathrm{Trial}^{73}$. In the NASCET trial, ${ }^{69}$ the risk of stroke in symptomatic patients was $26 \%$ over 2 years with aspirin, only $8 \%$ with surgery, an absolute risk reduction of approximately $9 \%$ per year. In asymptomatic patients, the risk was approximately $11 \%$ over a 5 -year period in both the $\mathrm{ACAS}^{72}$ and $\mathrm{ACST}^{73}$ studies, or approximately $2 \%$ annually. The benefit of surgery is to reduce this risk to approximately $1 \%$ annually. The morbidity-mortality in the NASCET study was $5.5 \%$; in ACAS, it was approximately $2.3 \%$. Nonetheless, it can be readily seen that carotid endarterectomy in a symp- tomatic patient carries a benefit within a few months of surgery, with number needed to treat to prevent one stroke in $\sim 8$; with asymptomatic carotid stenosis, the benefit takes 2 to 3 years to be appreciated, and the number needed to treat is $\sim 100$.

The recent SAPPHIRE (stenting and angioplasty with protection in patients at high risk for endarterectomy) and ARCHeR (Acculink for revascularization of carotids in high-risk patients) studies have found carotid artery stenting to compare favorably with carotid endarterectomy in patients at high risk for surgical revascularization. ${ }^{74}$ Carotid stenting in conjunction with appropriate antiplatelet therapy offers a less invasive alternative to carotid endarterectomy for patients at high surgical risk and those ineligible for surgery. Preprocedural care includes dual clopidogrel/aspirin therapy, given 48 hours in advance of the procedure; postprocedural care includes dual clopidogrel/aspirin therapy for the first 4 weeks, followed by indefinite aspirin therapy. For patients with current indications for carotid endarterectomy, the CREST trial will compare this surgery against angioplasty and stenting. Carotid endarterectomy remains the standard treatment for these patients.

The NSA rates carotid endarterectomy as appropriate in patients with $50 \%$ to $99 \%$ stenosis and surgical risk $\leq 6 \%$, and in patients with $70 \%$ to $99 \%$ stenosis with large ulcer and surgical risk $>6 \%$; carotid endarterectomy is rated as inappropriate or uncertain for all other stroke patients. ${ }^{29}$ The SORT criteria ${ }^{24}$ clearly warrant an "A" recommendation of carotid endarterectomy for symptomatic stenosis of $\geq 70 \%$, whereas carotid endarterectomy for stenosis of $<70 \%$ in a symptomatic patient or for $>60 \%$ stenosis in an asymptomatic patient would receive a "B" rating. Stenting is also given a "B" rating, with the possible exception of the "high risk" patients studied in the SAPPHIRE study. $^{74}$

\section{Conclusions}

Many evidence-based treatments are effective in preventing stroke. In primary prevention, smoking cessation, exercise, blood pressure management, and correction of hyperlipidemia with diet and use of HMG-CoA reductase inhibitors (statins) reduce the risk of stroke. Carotid endarterectomy in patients with high-grade, asymptomatic carotid ste- 
nosis prevents stroke, although the absolute risk reduction is small. Patients with atrial fibrillation benefit from warfarin therapy. Antiplatelet agents have shown limited evidence of primary stroke prevention, although the recent Women's Health Initiative report ${ }^{23}$ did suggest a role for aspirin in stroke prevention in middle aged and elderly women.

In secondary stroke prevention, blood pressure and cholesterol management are critically important, and drug therapy, as well as assistance with smoking cessation, should be initiated before discharge from the hospital. Carotid surgery and anticoagulation for atrial fibrillation also have greater efficacy in patients who have already suffered TIA or stroke symptoms, compared with asymptomatic people. Antiplatelet therapy also has greater stroke preventive effect in secondary prevention. Because $\mathrm{CAD}, \mathrm{PAD}$, and many ischemic strokes share a common atherothrombotic mechanism, patients who have experienced atherothrombotic stroke are exposed to a cross-risk for events in other vascular beds, especially the coronary arteries. This crossrisk extends over the long term and heightens the need for effective long-term treatment for stroke patients. There is an additional need to reduce the risk of a recurrent event caused by the plaque responsible for the index event(s).

With regard to specific antiplatelet agents, aspirin, aspirin + ER-dipyridamole, and clopidogrel are all recommended for secondary stroke prevention. Aspirin + ER-dipyridamole has the most statistically potent data for stroke prevention but has not shown benefit in the cross-risks for CAD and $\mathrm{PAD}$. It is therefore recommended most in patients without evidence of these generalized atherothrombotic conditions. Clopidogrel is more effective than aspirin in reducing the risk of recurrent ischemic stroke and other atherothrombotic events. Clopidogrel has a safety profile similar to aspirin, but is associated with fewer gastric and CNS adverse events, including gastrointestinal and intracranial hemorrhage. The combination of clopidogrel + aspirin has not been shown to be effective for the secondary prevention of ischemic stroke. Future studies, such as the SPS 3 and CHARISMA trials, will provide additional information on the benefits of adding clopidogrel to aspirin in at-risk populations, including those with ischemic stroke. In addition, the ongoing PRoFESS trial will provide comparative efficacy information on clopi- dogrel versus ASA + ER-dipyridamole in secondary stroke prevention.

\section{References}

1. American Heart Association. Heart disease and stroke statistic-2004 update. Report available online at: http://www.americanheart.org/downloadable/ heart/1079736729696HDSStats2004UpdateREV319-04.pdf. 2004.

2. Rosenberg C, Popelka GM. Post-stroke rehabilitation. A review of guidelines for patient management. Geriatrics 2000;55:75-81.

3. Spagnoli LG, Mauriello A, Sangiorgi G, et al. Extracranial thrombotically active carotid plaque as a risk factor for ischemic stroke. JAMA 2004;292: $1845-52$.

4. Vickrey BG, Rector TS, Wickstrom SL, et al. Occurrence of secondary ischemic events among persons with atherosclerotic vascular disease. Stroke 2002;33:901-6.

5. CAPRIE Steering Committee. A randomised, blinded, trial of clopidogrel versus aspirin in patients at risk of ischaemic events (CAPRIE). Lancet 1996; 348:1329-39.

6. Diener HC, Cunha L, Forbes C, et al. European Stroke Prevention Study. 2. Dipyramidole and acetylsalicylic acid in the secondary prevention of stroke. J Neurol Sci 1996;143:1-13.

7. Hardie K, Hankey GJ, Jamrozik K, Broadhurst RJ, Anderson C. Ten-year survival after first stroke in the Perth community stroke study. Stroke 2003;34: $1842-6$.

8. Gorelick PB. Stroke prevention. Arch Neurol 1995; 52:347-55.

9. Volpato S, Maraldi C, Blé A, et al. Prescription on antithrombotic therapy in older patients hospitalised for transient ischemic attack and ischemic stroke: the GIFA study. Stroke 2004;35:913-7.

10. Holloway RG, Benesch C, Rush SR. Stroke prevention: narrowing the evidence-practice gap. Neurology 2000;54:1899-906.

11. Arora S, Broderick SP, Frankel M, et al. Acute stroke care in the US: results from 4 pilot prototypes of the Paul Coverdell National Acute Stroke Registry. Stroke 2005;36:1232-40.

12. Ovbiagele B, Saver JL, Fredieu A, et al. PROTECT: a coordinated stroke treatment program to prevent recurrent thromboembolic events. Neurology 2004; 63:1217-22.

13. Criqui MH, Langer RD, Fronek A, et al. Mortality over a period of 10 years in patients with peripheral arterial disease. N Engl J Med 1992;326:381-6.

14. Ness J, Aronow WS. Prevalence of coexistence of coronary artery disease, ischemic stroke, and peripheral arterial disease in older persons, mean age 80 years, in an academic hospital-based geriatrics practice. J Am Geriatr Soc 1999;47:1255-6. 
15. Adams RJ, Chimowitz MI, Alpert JS, et al. Coronary risk evaluation in patients with transient ischemic attack and ischemic stroke: a scientific statement for healthcare professionals from the Stroke Council and the Council on Clinical Cardiology of the American Heart Association/American Stroke Association. Stroke 2003;34:2310-22.

16. Johnston SC, Gress DR, Browner WS, Sidney S. Short-term prognosis after emergency department diagnosis of TIA. JAMA 2000;284:2901-6.

17. Ridker PM, Cushman M, Stampfer MJ, et al. Inflammation, aspirin, and the risk of cardiovascular disease in apparently healthy men. N Engl J Med 1997;336: 973-9.

18. Di Napoli M, Papa F, and Bocola V. C-Reactive protein in ischemic stroke: an independent prognostic factor. Stroke 2001;32:917-24.

19. Marquardt L, Ruf A, Mansmann U, et al. Course of platelet activation markers after ischemic stroke. Stroke 2002;33:2570-4.

20. Lees KR, Bath PMW, Naylor AR. Secondary prevention of transient ischemic attack and stroke. BMJ 2000;320:991-4.

21. Goldstein LB, Adams R, Becker K, et al. Primary prevention of ischemic stroke. A statement for healthcare professionals from the stroke council of the American Heart Association. Circulation 2001; 103:163-82.

22. Steering Committee of the Physicians' Health Study Research Group. Final report on the aspirin component of the ongoing Physicians' Health Study. N Engl J Med 1989;321:129-35.

23. Ridker PM, Cook NR, Lee I-M, et al. A randomized trial of low-dose aspirin in the primary prevention of cardiovascular disease in women. N Engl J Med 2005;352:1293-304.

24. Ebell MH, Siwek J, Weiss BD, et al. Strength of recommendation taxonomy (SORT): a patient-centered approach to grading evidence in the medical literature. J Am Board Fam Pract 2004;17:59-67.

25. Pearson TA, Blair SN, Daniels SR, et al. AHA guidelines for primary prevention of cardiovascular disease and stroke: 2002 update. Circulation 2002; 106:388-91.

26. Kirshner HS. Medical prevention of stroke. South Med J 2003;96:354-8.

27. Ezekowitz JA, Straus SE, Majumdar SR, et al. Stroke: strategies for primary prevention. Am Fam Physician 2003;68:2379-86.

28. Albers GW, Amarenco P, Easton JD, Sacco RL, Teal P. Antithrombotic and thrombolytic therapy for ischemic stroke: the Seventh ACCP Conference on Antithrombotic and Thrombolytic Therapy. Chest 2004;126(Suppl 3):483S-512S.

29. Hanley D, Gorelick PB, Elliot WJ, et al. Determining the appropriateness of selected surgical and medical management options in recurrent stroke prevention: a guideline for primary care physicians from the
National Stroke Association Work Group on Recurrent Stroke Prevention. J Stroke Cerebrovasc Dis 2004;13:196-207.

30. Strauss S, Majumdar SR, McAlister FA. New evidence for stroke prevention. JAMA 2002;288:138895.

31. Gueyffier F, Boissel JP, Boutitie F, et al. Effect of antihypertensive treatment in patients having already suffered from stroke. Gathering the evidence. The INDANA (INdividual Data ANalysis of Antihypertensive intervention trials) Project Collaborators. Stroke 1997;28:2557-62.

32. Chobanian AV, Bakris GL, Black HR, et al. Seventh report of the Joint National Committee on Prevention, Detection, Evaluation, and Treatment of High Blood Pressure. Hypertension 2003;42:1206-52.

33. PROGRESS Collaborative Group. Randomised trial of a perindopril-based blood-pressure-lowering regimen among 6105 individuals with previous stroke or transient ischemic attack. Lancet 2001;358:1033-41.

34. Schrader J, Lüders S, Kulschewski A, et al. The ACCESS study: evaluation of acute candesartan cilexetil therapy in stroke survivors. Stroke 2003;34: 1699-703.

35. The Heart Outcomes Prevention Evaluation Study Investigators. Effects of an angiotensin-convertingenzyme inhibitor, ramipril, on cardiovascular events in high-risk patients. N Engl J Med 2000;342:14553.

36. Dahlof B, Devereux RB, Kjeldsen SE, et al. Cardiovascular morbidity and mortality in the Losartan Intervention for Endpoint reduction in hypertension study (LIFE): a randomized trial against atenolol. Lancet 2002;359:995-1003.

37. The ALLHAT Officers and Coordinators for the ALLHAT Collaborative Research Group. Major outcomes in high-risk hypertensive patients randomized to angiotensin-converting enzyme inhibitor or calcium channel blocker vs diuretic. The antihypertensive and lipid-lowering treatment to prevent heart attack trial (ALLHAT). JAMA 2002;288:2981-97.

38. Wright JT, Dunn JK, Cutler JA, et al. Outcomes in hypertensive black and non-black patients treated with chlorthalidone, amlodipine, and lisinopril. JAMA 2005;293:1595-608.

39. AHA/ASA. Statins after ischemic stroke and transient ischemic attack: an advisory statement from the Stroke Council, American Heart Association and American Stroke Association. Stroke 2002;35:1023.

40. . Julius S, Kjeldsen SE, Weber M, et al. Outcomes in hypertensive patients at high cardiovascular risk treated with regimens based on valsartan or amlodipine: the VALUE randomised trial. Lancet 2004; 363:2022-31.

41. Heart Protection Study Collaborative Group. Effects of cholesterol-lowering with simvastatin on stroke and other major vascular events in 20,536 
people with cerebrovascular disease or other highrisk conditions. Lancet 2004;363:757-67.

42. Heart Protection Study Collaborative Group. MRC/ BHF Heart protection study of cholesterol lowering with simvastatin in 20536 high-risk individuals: a randomised placebo-controlled trial. Lancet 2002; 360:7-22.

43. The SPARCL Investigators. Design of the Stroke Prevention by Aggressive Reduction in Cholesterol Levels (SPARCL) study. Proceedings of the Ongoing Clinical Trials Session, 28th International Stroke Conference. 2003. Phoenix, Arizona.

44. Atrial Fibrillation Investigators. Risk factors for stroke and efficacy of antithrombotic therapy in atrial fibrillation. Analysis of pooled data from five randomized controlled trials. Arch Intern Med 1994; 154:1449-57.

45. EAFT (European Atrial Fibrillation Trial) Study Group. Secondary prevention in non-rheumatic atrial fibrillation after transient ischemic attack of minor stroke. Lancet 1993;342:1255-62.

46. Olsson SB, Executive Steering Committee on behalf of the SPORTIF III Investigators. Stroke prevention with the oral direct thrombin inhibitor ximelagatran compared with warfarin in patients with non-valvular atrial fibrillation (SPORTIF III): randomised controlled trial. Lancet 2003;362:1691-8.

47. Albers GW, Diener HC, Frison L, et al. Ximelagatran vs warfarin for stroke prevention in patients with nonvalvular atrial fibrillation. A randomized trial. JAMA 2005;293:690-8.

48. SPIRIT. A randomized trial of anticoagulants versus aspirin after cerebral ischemia of presumed arterial origin. The Stroke Prevention in Reversible Ischemia Trial (SPIRIT) Study Group. Ann Neurol 1997;42:857-65.

49. Mohr JP, Thompson JLP, Lazar RM, et al. A comparison of warfarin and aspirin for the prevention of recurrent ischemic stroke. N Engl J Med 2001;345: $1444-51$.

50. Levine SR, Brey RL, Tilley BC, et al. Antiphospholipid antibodies and subsequent thrombo-occlusive events in patients with ischemic stroke. JAMA 2004; 291:576-84.

51. Homma S, Sacco RL, Di Tullio MR, et al. Effect of medical treatment in stroke patients with patent foramen ovale: patent foramen ovale in Cryptogenic Stroke Study. Circulation 2002;105:2625-31.

52. Chimowitz MI, Lynn MJ, Howlett-Smith H, et al. Comparison of warfarin and aspirin for symptomatic intracranial arterial stenosis. N Engl J Med 2005; 352:1305-16.

53. Homma S, Di Tullio MR, Sciacca RR, et al. Effect of aspirin and warfarin therapy in stroke patients with valvular strands. Stroke 2004;35:1436-42.

54. Alberts MJ, Atkinson R. Risk reduction strategies in ischemic stroke. The role of antiplatelet therapy. Clin Drug Invest 2004;24:245-54.
55. Antithrombotic Trialists' Collaboration. Collaborative meta-analysis of randomised trials of antiplatelet therapy for prevention of death, myocardial infarction, and stroke in high risk patients. BMJ 2002;324: 71-86.

56. Weisman SM, Graham D. Evaluation of the benefits and risks of low-dose aspirin in the secondary prevention of cardiovascular and cerebrovascular events. Arch Intern Med 2002;162:2197-202.

57. Sacco RL, Sivenius J, Diener H-C. Efficacy of aspirin plus extended-release dipyridamole in preventing recurrent stroke in high-risk populations. Arch Neurol 2005;62:403-8.

58. Bhatt DL, Marso SP, Hirsch AT, et al. Amplified benefit of clopidogrel versus aspirin in patients with diabetes mellitus. Am J Cardiol 2002;90:625-8.

59. Bhatt DL, Chew DP, Hirsch AT, et al. Superiority of clopidogrel versus aspirin in patients with prior cardiac surgery. Circulation 2001;103:363-8.

60. Ringleb PA, Bhatt DL, Hirsch AT, et al. Benefit of clopidogrel over aspirin is amplified in patients with a history of ischemic events. Stroke 2004;35:528-32.

61. Diener HC, Bogousslavsky J, Brass LM, et al. Aspirin and clopidogrel compared with clopidogrel alone after recent ischaemic stroke or transient ischaemic attack in high-risk patients (MATCH): randomised, double-blind, placebo-controlled trial. Lancet 2004; 364:331-7.

62. Amarenco P, Donnan GA. Should the MATCH results be extrapolated to all stroke patients and affect ongoing trials evaluating clopidogrel plus aspirin? Stroke 2004;35:2606-8.

63. Markus HS, Ringelstein EB, for the CARESS Investigators. The effect of dual antiplatelet therapy compared with aspirin on asymptomatic embolisation in carotid stenosis: the CARESS trial. Cerebrovasc Dis 2004;17(Suppl 5):39.

64. The Clopidogrel in Unstable Angina to Prevent Recurrent Events Trial Investigators. Effects of clopidogrel in addition to aspirin in patients with acute coronary syndromes without ST-segment elevation. N Engl J Med 2001;345:494-502.

65. Pirmohamed M, James S, Meakin S, et al. Adverse drug reactions as cause of admission to hospital: prospective analysis of 18820 patients. BMJ 2004; 329:15-9.

66. Majhail NS, Lichtin AE. Clopidogrel and thrombotic thrombocytopenic purpura: no clear case for causality. Cleve Clin J Med 2003;70:466-70.

67. Peters RJG, Mehta SR, Fox KAA, et al. Effects of aspirin dose when used alone or in combination with clopidogrel in patients with acute coronary syndromes. Observations from the Clopidogrel in Unstable angina to prevent Recurrent Events (CURE) Study. Circulation 2003;108:1682-7.

68. Steinhubl SR, Berger PB, Mann JT, 3rd, et al. Early and sustained dual oral antiplatelet therapy following 
percutaneous coronary intervention: a randomized controlled trial. JAMA 2002;288:2411-20.

69. North American Symptomatic Carotid Endarterectomy Trial Collaborators. Beneficial effect of carotid endarterectomy in symptomatic patients with highgrade carotid stenosis. N Engl J Med 1991;325:44553.

70. Mayberg MR, Wilson SE, Yatsu F, et al. Carotid endarterectomy and prevention of cerebral ischemia in symptomatic carotid stenosis. Veterans Affairs Cooperative Studies Program 309 Trialist Group. JAMA 1991;266:3289-94.

71. European Carotid Surgery Trialists' Collaborative Group. Endarterectomy for moderate symptomatic carotid stenosis: interim results from the MRC Eu- ropean Carotid Surgery Trial. Lancet 1991;337: 1591-3.

72. Executive Committee for the Asymptomatic Carotid Atherosclerosis Study. Endarterectomy for asymptomatic carotid artery stenosis. JAMA 1995;273: 1421-8.

73. MRC Asymptomatic Carotid Surgery Trial (ACST) Collaborative Group. Prevention of disabling and fatal strokes by successful carotid endarterectomy in patients without recent neurological symptoms: randomized controlled trial. Lancet 2004;363:1491502.

74. Yadav JS, Wholey MH, Kuntz RE, et al. Protected carotid-artery stenting versus endarterectomy in high-risk patients. N Engl J Med 2004;351:1493501. 